\title{
THE EXTENSION OF WEAKLY P3 PROPERTIES AND THE INTERNALIZATION OF THE WEAKLY $P($ URYSOHN) AND WEAKLY P3 PROCESSES
}

\section{CHARLES DORSETT}

Department of Mathematics

Texas A\&M University-Commerce

Texas 75429

USA

e-mail: charles.dorsett@tamuc.edu

\begin{abstract}
In this paper, the investigation and use of $T_{0}$-identification spaces continue with the extension of known weakly $P 3$ properties and each of the weakly $P($ Urysohn) and weakly $P 3$ processes are internalized.
\end{abstract}

\section{Introduction and Preliminaries}

The regular and $T_{3}$ separation axioms were introduced in 1921 [22].

Definition 1.1. A space $(X, T)$ is regular iff for each closed set $C$ and each $x \notin C$, there exist disjoint open sets $U$ and $V$ such that $x \in U$ and $C \subseteq V$. A regular $T_{1}$ space is denoted by $T_{3}$.

2010 Mathematics Subject Classification: 54A05, 54B15, 54D10.

Keywords and phrases: $T_{0}$-identification spaces, weakly $P$ properties, separation axioms.

Received June 30, 2018

(C) 2018 Scientific Advances Publishers 
Each of regular and $T_{3}$ are useful, important axioms greatly studied and used in mathematics. In this paper, regular and $T_{3}$ are further investigated within the continued study of weakly $P 3$ spaces and properties.

Weakly $P 3$ is one of several recently defined properties motivated by past use of $T_{0}$-identification spaces, which were introduced in 1936 [20].

Definition 1.2. Let $(X, T)$ be a space, let $R$ be the equivalence relation on $X$ defined by $x R y$ iff $C l(\{x\})=C l(\{y\})$, let $X_{0}$ be the set of $R$ equivalence classes of $X$, let $N: X \rightarrow X_{0}$ be the natural map, and let $Q(X, T)$ be the decomposition topology on $X_{0}$ determined by $(X, T)$ and the natural map $N$. Then $\left(X_{0}, Q(X, T)\right)$ is the $T_{0}$-identification space of $(X, T)$.

In the 1936 paper [20], it was shown that a space is pseudometrizable iff its $T_{0}$-identification space is metrizable. $T_{0}$-identification spaces were cleverly created to obtain for a space $(X, T)$ a strongly $(X, T)$ related $T_{0}$-identification space with the $T_{0}$ separation axiom added, making $T_{0}$-identification spaces a useful tool in the continued study of mathematics.

In the 1975 paper [18], weakly Hausdorff and Hausdorff were jointly characterized using $T_{0}$-identification spaces: A space is weakly Hausdorff iff its $T_{0}$-identification space is Hausdorff. Within the 1975 paper [18], it was shown that weakly Hausdorff is equivalent to the $R_{1}$ separation axiom introduced in 1961 [1]. A space $(X, T)$ is $R_{1}$ iff for $x$ and $y$ in $X$ such that $C l(\{x\}) \neq C l(\{y\})$, there exist disjoint open sets $U$ and $V$ such that $x \in U$ and $y \in V$. 
Within the 1961 paper [1], the $R_{1}$ separation axiom was used to further characterize $T_{2}$. A space is $T_{2}$ iff it is $\left(R_{1}\right.$ and $\left.T_{1}\right)$. Also, in the 1961 paper [1], the $R_{0}$ separation axiom was rediscovered and used to further characterize $T_{1}$. A space is $R_{0}$ iff for each closed set $C$ and each $x \notin C, C \cap C l(\{x\})=\phi$ [19]. A space is $T_{1}$ iff it is $\left(R_{0}\right.$ and $\left.T_{0}\right)$ [1].

Using the characterizations of pseudometrizable and $R_{1}$ given above as motivation and model, weakly Po was defined in 2015 [2].

Definition 1.3. Let $P$ be a topological property for which $P_{o}=$ ( $P$ and $\left.T_{0}\right)$ exists. Then $(X, T)$ is weakly $P o$ iff its $T_{0}$-identification space $\left(X_{0}, Q(X, T)\right)$ has property $P$. A topological property $P o$ for which weakly $P o$ exists is called a weakly $P o$ property.

Since, as given above, $T_{0}$-identification spaces are $T_{0}$, then a space is weakly $P o$ iff its $T_{0}$-identification space is $P_{o}$. The study of weakly $P_{o}$ spaces and properties has proved to be an extremely productive, revealing study, which has already changed the study of topology forever. A major breakthrough in the study of weakly Po properties occurred when it was shown that $\{Q o \mid Q$ is a topological property for which weakly $Q o$ exists $\}=\{Q o \mid Q$ is a topological property and Qo exists $\}$ [3]. As would be required, within that paper a topological property $S$ was shown to exist such that $S=$ weakly $Q o$ and $S o=Q o[3]$.

The search for a topological property that failed to be weakly Po lead to the need and use of "not- $T_{0}$ ", revealing "not- $T_{0}$ " as a powerful, useful tool in the study of topology, motivating the inclusion of "not- $T_{0}$ " and "not- $P$ ", where $P$ is a topological property for which "not- $P$ " exists, as powerful, useful properties and tools for investigation and use in the study of topology. The use of those new properties in a 2016 paper [4] quickly and easily revealed a never before imagined topological property that has changed the study of topology forever: $L=\left(T_{0}\right.$ or "not- $T_{0}$ " $)=$ ( $P$ or "not- $P$ "), where $P$ is a topological property for which "not- $P$ " exists, is the least of all topological properties. 
In the study of weakly $P o$ spaces and properties, it was shown that $R_{0}=$ weakly $\left(R_{0}\right) o=$ weakly $T_{1}$ and $R_{1}=$ weakly $\left(R_{1}\right) o=$ weakly $T_{2}$, and for a topological property $P$ for which weakly $P o$ exists, (weakly $P o$ ) $O$ $=P o$ [2]. The existence of the least of all topological properties $L$ raised the questions of whether there is a least topological property, which together with $T_{i}$, equals $T_{i+1}, i=0,1$, or more generally, is there a least topological property, which together with $T_{0}$, equals $P_{o}$ for a weakly $P_{o}$ property. The answer once again showed "not- $T_{0}$ " to be a powerful, useful, foundational property in the study of topology: For a weakly Po property $Q o$, the least topological property, which together with $T_{0}$, equals $Q o$ is ((weakly Qo) or "not- $T_{0}$ ") [5]. Hence $\left(R_{i}\right.$ or "not- $T_{0}$ ") is the least topological property, which together with $T_{0}$, equals $T_{i+1}, i=0,1$.

The study of $T_{0}$-identification spaces continued with the introduction and investigation of $T_{0}$-identification $P$ [6], weakly $P 1$ [7], weakly $P 2$ [8], weakly $P($ Urysohn) [9], and weakly $P 3$ [10] spaces and properties.

Definition 1.4. Let $S$ be a topological property. Then $S$ is a $T_{0}$-identification $P$ property iff $S$ is simultaneously shared by a space and its $T_{0}$-identification space [6].

Definition 1.5. Let $P$ be a topological property for which $P 1=$ $\left(P\right.$ and $\left.T_{1}\right)$ exists. Then a space $(X, T)$ is weakly $P 1$ iff its $T_{0}$-identification space $\left(X_{0}, Q(X, T)\right)$ is $P 1$. A topological property $P 1$ for which weakly $P 1$ exists is called a weakly $P 1$ property [7].

Definition 1.6. Let $P$ be a topological property for which $P 2=$ $\left(P\right.$ and $\left.T_{2}\right)$ exists. Then a space $(X, T)$ is weakly $P 2$ iff its $T_{0}$-identification space $\left(X_{0}, Q(X, T)\right)$ is $P 2$. A topological property $P 2$ for which weakly $P 2$ exists is called a weakly $P 2$ property [8]. 
Urysohn spaces were introduced in 1925 [21].

Definition 1.7. A space $(X, T)$ is Urysohn iff for distinct elements $x$ and $y$ in $X$, there exist open sets $U$ and $V$ such that $x \in U, y \in V$, and $C l(U) \cap C l(V)=\phi$.

Definition 1.8. Let $P$ be a topological property for which $P$ (Urysohn) $=(P$ and Urysohn $)$ exists. Then a space $(X, T)$ is weakly $P($ Urysohn $)$ iff its $T_{0}$-identification space $\left(X_{0}, Q(X, T)\right)$ is $P($ Urysohn). A topological property $Q$ (Urysohn) for which weakly $Q$ (Urysohn) exists is called a weakly $P($ Urysohn) property [9].

In the 1988 paper [11], using weakly Hausdorff $=R_{1}$ as a model, weakly Urysohn spaces were defined.

Definition 1.9. A space $(X, T)$ is weakly Urysohn iff for $x$ and $y$ in $X$ for which $C l(\{x\}) \neq C l(\{y\})$, there exist open sets $U$ and $V$ such that $x \in U, y \in V$, and $C l(U) \cap C l(V)=\phi$.

In the paper [9], it was shown that (weakly Urysohn) = weakly (weakly Urysohn)o = weakly Urysohn.

Definition 1.10. Let $Q$ be a topological property for which $Q 3=$ $\left(Q\right.$ and $\left.T_{3}\right)$ exists. Then a space is weakly $Q 3$ iff its $T_{0}$-identification space is $Q 3$. A topological property $Q 3$ for which weakly $Q 3$ exists is called a weakly $P 3$ property [10].

Below, the investigation of weakly $P 3$ spaces and properties continues.

\section{Basic Properties for Weakly P3 Spaces}

Within the paper [10], it was unknown which topological properties were weakly $P 3$ properties and basic properties of weakly $P 3$ spaces were investigated under the requirement that weakly $P 3$ existed. Below a 
basic property for $P 3$, where $P 3$ exists, is combined with the complete characterization of weakly $P o$ properties given above to completely characterize weakly $P 3$ properties, which is then used to extend all previously known weakly $P 3$ properties to all topological properties $P$ for which $P 3$ exists.

Theorem 2.1. Let $Q$ be a topological property for which $Q 3$ exists. Then $(Q 3)_{o}=Q 3$.

Proof. Since $T_{3}$ implies $T_{0}$, then $(Q 3) o=\left(\left(Q\right.\right.$ and $\left.T_{3}\right)$ and $\left.T_{0}\right)=$ $\left(Q\right.$ and $\left(T_{3}\right.$ and $\left.\left.T_{0}\right)\right)=\left(Q\right.$ and $\left.T_{3}\right)=Q 3$.

Theorem 2.2. $A=\{Q 3 \mid Q$ is a topological property and weakly $Q 3$ exists $\}=B=\{Q 3 \mid Q$ is a topological property and $Q 3$ exists $\}$.

Proof. Let $Q 3 \in A$. Then, by definition, $Q 3 \in B$.

Conversely, suppose $Q 3 \in B$. Then $Q 3=(Q 3)_{o}$ is a weakly $P o$ property and weakly $(Q 3) o$ exists. Hence weakly $Q 3=$ weakly $(Q 3) o$ exists and $Q 3 \in A$. Hence $A=B$.

In the same manner, it was shown that

$\{Q p \mid Q$ is a topological property and weakly $Q p$ exists $\}=$

$\{Q p \mid Q$ is a topological property and $Q p$ exists $\}, p=1$ [12], $p=2$ [13], and $p=$ Urysohn [14].

In mathematics, one strategy for solving a new problem is to reduce the new problem to an already solved problem, if possible, and then use the solution of the already solved problem to solve the new problem. When successful, the strategy is particularly pleasing. Above, the fact that $(Q 3) o=Q 3$ allowed questions concerning weakly $Q 3$ to be resolved by using known properties of weakly $Q o$. Below use of $Q 3=(Q 3) o$ and the same strategy will be used to extend previously known properties of weakly $P 3$ under the requirement that weakly $P 3$ exists to all topological properties for which $P 3$ exists and to answer other basic questions concerning weakly $Q 3$ spaces and properties. 
Theorem 2.3. Let $Q$ be a topological property for which $P 3$ exists. Then weakly $Q 3$ is a unique topological property.

Proof. Since $(Q 3)_{o}=Q 3$, then $Q 3$ is a weakly Po property. Since weakly $P_{0}$ is a unique topological property [2], then weakly $Q 3$ is a unique topological property.

A very basic question to ask concerning weakly $Q 3$ is how are weakly $Q 3$ spaces and properties related to the earlier weakly defined properties, which is addressed below.

Theorem 2.4. Let $Q$ be a topological property for which $Q 3$ exists. Then weakly Q3 implies weakly Q(Urysohn), which implies weakly Q2, which implies weakly Q1, which implies weakly Qo.

Proof. Since $Q 3$ exists and $Q 3$ implies $Q$ (Urysohn), then $Q$ (Urysohn) exists and weakly $Q($ Urysohn) exists. Let $(X, T)$ be a space that is weakly $Q 3$. Then $\left(X_{0}, Q(X, T)\right)$ has property $Q 3$, which implies $\left(X_{0}, Q\right.$ $(X, T))$ has property $Q$ (Urysohn) and $(X, T)$ is weakly $Q$ (Urysohn). Hence weakly $Q 3$ implies weakly $Q($ Urysohn). The remainder of the proof is similar and omitted.

Within the paper [15], it was shown that that if $Q$ (Urysohn) exists, then weakly $Q($ Urysohn $)=(($ weakly $Q 2)$ and (weakly Urysohn) $)=$ $(($ weakly $Q 1)$ and (weakly Urysohn)) $=$ ((weakly Qo) and (weakly Urysohn)), which is used below.

Theorem 2.5. Let $Q$ be a topological property for which $Q 3$ exists. Then weakly $Q 3=(($ weakly $Q($ Urysohn $))$ and regular $)=(($ weakly $Q 2)$ and regular $)=(($ weakly $Q 1)$ and regular $)=(($ weakly $Q o)$ and regular $)$.

Proof. Let $(X, T)$ be weakly $Q 3$. Since $T_{3}$ implies Urysohn, then $\left(X_{0}, Q(X, T)\right)$ is $Q 3=\left(Q\right.$ and $\left.T_{3}\right)=\left(Q\right.$ and (Urysohn and $\left.\left.T_{3}\right)\right)=((Q$ and Urysohn $)$ and $\left.T_{3}\right)=\left(Q(\right.$ Urysohn $)$ and $\left.T_{3}\right)$. Since $\left(X_{0}, Q(X, T)\right)$ is $Q$ (Urysohn), then $(X, T)$ is weakly $Q\left(\right.$ Urysohn) and since $\left(X_{0}, Q(X, T)\right)$ is 
$T_{3}$, then $(X, T)$ is regular [10]. Hence $(X, T)$ is ((weakly $Q$ (Urysohn)) and regular) and weakly $Q 3$ implies (weakly $Q$ (Urysohn)) and regular). Since regular implies (weakly Urysohn), then ((weakly $Q$ (Urysohn)) and regular $)=((($ weakly $Q 2)$ and (weakly Urysohn $))$ and regular $)=(($ weakly Q2) and ((weakly Urysohn) and regular $))=(($ weakly Q2) and regular). Similarly, ((weakly $Q($ Urysohn $))$ and regular $)=(($ weakly $Q 1)$ and regular $)$ $=$ ((weakly $Q o$ and regular). Thus weakly Q3 implies ((weakly $Q($ Urysohn $))$ and regular $)=(($ weakly $Q 2)$ and regular $)=(($ weakly $Q 1)$ and regular $)=\left((\right.$ weakly $Q o$ and regular $)$ and $(X, T)$ is ((weakly $Q_{o}$ and regular). Then $\left(X_{0}, Q(X, T)\right)$ is $\left(Q o\right.$ and $\left.T_{3}\right)=\left(Q\right.$ and $\left.T_{3}\right)=Q 3$ and $(X, T)$ is weakly $Q 3$. Hence weakly $Q 3=(($ weakly $Q($ Urysohn $))$ and regular $)=(($ weakly $Q 2)$ and regular $)=(($ weakly $Q 1)$ and regular $)=$ ((weakly $Q o)$ and regular).

Theorem 2.6. Let $Q$ be a topological property for which $Q 3$ exists. Then (weakly $Q 3) o=Q 3$.

Proof. Since $Q 3=(Q 3) o$, then (weakly Q3) $o=($ weakly $(Q 3) o) o=(Q 3) o$ $[2]=Q 3$. Within the paper [2], it was shown that neither $T_{0}$ nor "not- $T_{0}$ " are weakly Qo. Combining this result with Theorem 2.1 gives the next result.

Corollary 2.1. Neither $T_{0}$ nor "not- $T_{0}$ " are weakly $Q 3$.

Theorem 2.7. Let $Q$ be a topological property for which $Q 3$ exists. Then weakly $\mathrm{Q} 3=(\mathrm{Q} 3$ or ((weakly Q3) and "not-T,")), where both Q3 and ((weakly Q3 and "not-T,")) exist and weaker than weakly Q3, and neither are weakly P3.

Proof. Since weakly $Q 3=(($ weakly Q3) and $L)=(($ weakly Q3) and $\left(T_{0}\right.$ or "not- $T_{0}$ ") $)=\left(\left(\right.\right.$ weakly Q3)o or $\left(\left(\right.\right.$ weakly Q3) and "not- $T_{0}$ ") $=$ (Q3 or ((weakly Q3) and "not- $T_{0}$ ")) and weakly $Q 3$ is neither $T_{0}$ nor "not- $T_{0}$ ", then both $Q 3$ and ((weakly Q3) and "not- $T_{0}$ ") exist and neither are weakly $P 3$. 
Theorem 2.8. Let $Q$ be a topological property for which $Q 3$ exists. Then $($ weakly Q3)o $=($ weakly Q3)1 $=($ weakly Q3 $) 2=($ weakly Q3) $($ Urysohn $)=($ weakly Q3) $3=Q 3$.

Proof. Since (weakly Q3) $3=\left(\left(\right.\right.$ weakly Q3) and $\left.T_{3}\right)=(($ weakly Q3) $)$ and $\left(T_{0}\right.$ and $\left.T_{3}\right)=\left(\left((\right.\right.$ weakly $Q 3)$ and $\left.T_{0}\right)$ and $\left.T_{3}\right)=\left((\right.$ weakly $Q 3) o$ and $\left.T_{3}\right)$ $=\left(Q 3\right.$ and $\left.T_{3}\right)=(Q 3) 3=Q 3$, then (weakly Q3)3=Q3. Since (weakly Q3) $($ Urysohn $)=\left(\left(\right.\right.$ weakly Q3) and $\left(T_{0}\right.$ and Urysohn $\left.)\right)=(($ weakly Q3 $) o$ and $)$ $($ Urysohn $))=(Q 3)$ and $($ Urysohn $))=\left(Q\right.$ and $\left(T_{3}\right.$ and $($ Urysohn $\left.\left.)\right)\right)=(Q$ and $\left.T_{3}\right)=Q 3$, then (weakly Q3) $($ Urysohn $)=Q 3$. In a similar manner, which is omitted, the remainder of the theorem is true.

Another fundamental question is what happens when the weakly Q3 process is repeated. Below the fact that (weakly Q3) $3=Q 3$ is used to quickly resolve the question.

Corollary 2.2. Let $Q$ be a topological property for which $Q 3$ exists. Then weakly (weakly Q3)3 = weakly Q3.

Also, use of the results above quickly and easily give the following property.

Corollary 2.3. Let $Q$ be a topological property for which $Q 3$ exists. Then the least topological property, which together with $T_{0}$, equals $Q 3$ is ((weakly Q3) or “not-T $T_{0}$ ).

Within the paper [4], it was shown that for a topological property $Q$ for which weakly $Q o$ exists, weakly $Q o$ is a $T_{0}$-identification $P$ property. Use of the complete characterization of weakly Po properties above, the property can be restated for each topological property $Q$ for which $Q o$ exists, which is used below. 
Theorem 2.9. Let $Q$ be a topological property for which $Q 3$ exists and let $(X, T)$ be a space. Then $(X, T)$ is weakly $Q 3$ iff $\left(X_{0}, Q(X, T)\right)$ is weakly $Q 3$, i.e., weakly $Q 3$ is a $T_{0}$-identification $P$ property and there exists a topological property $J$ such that $J=$ weakly $Q 3$ and $J o=Q 3$.

Proof. Since, as given above, weakly $P o$ is a $T_{0}$-identification $P$ property, then weakly $Q 3=$ weakly $(Q 3) o$ is a $T_{0}$-identification $P$ property. Since $Q 3=(Q 3) o$, then, by the results above, there exists a topological property $J$ such that $J=$ weakly $(Q 3) o$ and $J o=(Q 3) o$. Thus $J$ is a topological property for which $J=$ weakly $Q 3$ and $J o=Q 3$.

Theorem 2.10. Let $P$ and $Q$ be topological properties such that $P 3$ and $Q 3$ exist. Then weakly $P 3=$ weakly $Q 3$ iff $P 3=Q 3$.

Proof. Suppose weakly $P 3=$ weakly $Q 3$. Then weakly $(P 3) o=$ weakly $(Q 3) o$ and $P 3=(P 3)_{o}=(Q 3) o[6]=Q 3$.

Conversely suppose $P 3=Q 3$. Then weakly $(P 3)_{o}=$ weakly $(Q 3) o$ and weakly $(P 3) o=$ weakly $(Q 3) o[6]$ and weakly $P 3=$ weakly $Q 3$.

Theorem 2.11. Let $P$ and $Q$ be topological properties such that each of $P 3$ and $Q 3$ exist. Then weakly P3 implies weakly Q3 iff P3 implies Q3.

Proof. Since weakly $(P 3)_{o}$ implies weakly $(Q 3) o$ iff $(P 3) o$ implies (Q3)o [17], then weakly $P 3$ implies weakly $Q 3$ iff $P 3$ implies $Q 3$.

\section{Equivalent Separation Axioms and the Internalization Processes}

Theorem 3.1. Let $Q$ be a topological property for which $Q 3$ exists. Then within each weakly $Q 3$ space $(X, T)$, all of $T_{3}$, Urysohn, $T_{2}, T_{1}$, and $T_{0}$ are equivalent. 
Proof. Since it is always true that $T_{3}$ implies Urysohn, which implies $T_{2}$, which implies $T_{1}$, which implies $T_{0}$, then the implications are true in $(X, T)$. Suppose $(X, T)$ is $T_{0}$. Then $(X, T)$ is (weakly $Q 3$ and $\left.T_{0}\right)=($ weakly $Q 3) o=Q 3$, which implies $T_{3}$.

Corollary 3.1. Let $Q$ be a topological property for which Q3 exists. Then within each weakly Q3 space $(X, T)$, all of "not-T,", "not-Urysohn", “not- $T_{2}$ ", "not- $T_{1}$ ", and "not- $T_{0}$ ” are equivalent.

Combining Corollary 3.1 with Theorem 2.7 gives the following results.

Corollary 3.2. Let $Q$ be a topological property for which $Q 3$ exists. Then weakly $Q 3=\left(Q 3\right.$ or $\left((\right.$ weakly $Q 3)$ and "not- $T_{1}$ ”), where both Q3 and ((weakly Q3 and “not-T,")) exist and weaker than weakly Q3, and neither are weakly $P 3$, weakly $Q 3=\left(Q 3\right.$ or ((weakly Q3) and "not- $T_{2}$ ")), where both Q3 and ((weakly Q3 and "not- $T_{2}$ ”)) exist and weaker than weakly Q3, and neither are weakly P3, weakly $Q 3=(Q 3$ or ((weakly Q3) and "not-Urysohn")), where both Q3 and ((weakly Q3 and "notUrysohn")) exist and weaker than weakly Q3, and neither are weakly P3, and weakly $Q 3=\left(Q 3\right.$ or $\left(\left(\right.\right.$ weakly Q3) and "not- $T_{3}$ ”)), where both Q3 and ((weakly Q3 and “not-T,")) exist and weaker than weakly Q3, and neither are weakly P3.

In the same manner, $T_{0}$ in Corollary 2.3 can be replaced by each of $T_{1}, T_{2}$, Urysohn, and $T_{3}$ and "not- $T_{0}$ " can be replaced by each of "not- $T_{1}$ ", "not- $T_{2}$ ", "not-Urysohn", and "not- $T_{3}$ ”.

Thus, as documented above, $T_{0}$-identification spaces are far more powerful and useful than ever imagined before the 2015 paper [2] and the investigations of weakly $P$ spaces and properties given above have not only revealed many never before imagined properties changing the study 
of topology forever, but, also, provided needed tools to resolve many unanswered, basic, foundational questions within topology. However, when using $T_{0}$-identification spaces and weakly $P$ spaces and properties, there is, by definition, a constant transition between a space and its $T_{0}$-identification space, which is tedious and sometimes unclear, raising the question of whether the $T_{0}$-identification space process and weakly $P$ processes could somehow be internalized in the initial space making each process simpler and more understandable. The investigation of that question led to the introduction and use of OXTO subsets and OXTO subspaces for a space $(X, T)[16]$.

Definition 3.1. Let $(X, T)$ be a space and for each $x \in X$, let $C_{x}$ be the $T_{0}$-identification equivalence class containing $x$. Then $Y$ is an OXTO subset of $X$ iff $Y$ contains exactly one element from each equivalence class $C_{x}$ and $\left(Y, T_{Y}\right)$ is called an OXTO subspace of $(X, T)$ [16].

Within the paper [16], it was shown that for a space $(X, T)$, for each OXTO subset $Y$ of $X,\left(Y, T_{Y}\right)$ is homeomorphic to $\left(X_{0}, Q(X, T)\right)$ and thus $T_{0}$. Also, within that paper it was shown that for each topological property $Q$ for which $Q o$ exists, a space is weakly $Q o$ iff for each $O X T O$ subset $Y$ of $X,\left(Y, T_{Y}\right)$ has property $Q o$, which can be, and has been, used to precisely determine weakly $Q_{o}[16]$. Thus, the $T_{0}$-identification space and weakly $P o$ processes are completely internalized by the use of OXTO subsets and subspaces for a space $(X, T)$.

In the same manner, within the paper [12], the weakly $P 1$ process was completely internalized by the use of OXTO subsets and subspaces for a space $(X, T)$ and within the paper [13], the weakly $P 2$ process was completely internalized by the use of OXTO subsets and subspaces for a space $(X, T)$. Below each of the weakly $P($ Urysohn) and weakly $P 3$ processes are internalized. 
Theorem 3.2. Let $Q$ be a topological property for which $Q($ Urysohn) exists. Then for a space $(X, T),(X, T)$ is weakly Q(Urysohn) iff for each OXTO subset $Y$ of $X,\left(Y, T_{Y}\right)$ is Q(Urysohn).

Proof. Suppose $(X, T)$ is weakly $Q($ Urysohn). Then $(X, T)$ is weakly $(Q(\text { Urysohn }))_{O}$ and, by the results above, for each OXTO subset $Y$ of $(X, T),\left(Y, T_{Y}\right)$ is $(Q($ Urysohn $)) o=Q($ Urysohn $)$.

Conversely, suppose that for a space $(X, T)$, for each $O X T O$ subset $Y$ of $(X, T),\left(Y, T_{Y}\right)$ is $Q$ (Urysohn). Then for each OXTO subset $Y$ of $(X, T),\left(Y, T_{Y}\right)$ is $(Q($ Urysohn $)) o$ and, by the results above, $(X, T)$ is weakly $(Q($ Urysohn $)) o=$ weakly $Q($ Urysohn $)$.

Theorem 3.3. Let $Q$ be a topological property for which $Q 3$ exists. Then for a space $(X, T),(X, T)$ is weakly Q3 iff for each OXTO subset $Y$ of $X,\left(Y, T_{Y}\right)$ is $Q 3$.

Proof. Suppose $(X, T)$ is weakly $Q 3$. Then $(X, T)$ is weakly $(Q 3) o$ and, by the results above, for each OXTO subset $Y$ of $(X, T),\left(Y, T_{Y}\right)$ is $(Q 3) o=Q 3$.

Conversely, suppose that for a space $(X, T)$, for each $O X T O$ subset $Y$ of $(X, T),\left(Y, T_{Y}\right)$ is $Q 3$. Then for each OXTO subset $Y$ of $(X, T),\left(Y, T_{Y}\right)$ is $(Q 3) o$ and, by the results above, $(X, T)$ is weakly $(Q 3) o=$ weakly $Q 3$.

Thus, the weakly $P($ Urysohn) and weakly $P 3$ processes have been completely internalized by the use of OXTO subsets and subspaces for a space $(X, T)$.

The continued investigation of weakly $P o$ spaces and properties using OXTO subsets and subspaces for a space $(X, T)$ led to an unexpected discovery [17]. 
Definition 3.2. Let $(X, T)$ be a space, let $Y$ be a subspace of $(X, T)$, and let $Q$ be a topological property for which $Q o$ exists. Then $\left(Y, T_{Y}\right)$ is a maximal, proper, dense, Qo subspace of $(X, T)$ iff $\left(Y, T_{Y}\right)$ is a proper, dense, $Q o$ subspace of $(X, T)$ such that for each subspace $\left(Z, T_{Z}\right)$ of $X$, where $Z$ properly contains $Y,\left(Z, T_{Z}\right)$ is "not-Qo" [17].

Result 3.1. Let $(X, T)$ be a space and let $Q$ be a topological property for which $Q o$ exists. Then for each $O X T O$ subset $Y$ of $X,\left(Y, T_{Y}\right)$ is a maximal, proper, dense, Qo subspace of $(X, T)$ iff $(X, T)$ is (weakly Qo and "not- $T_{0}$ ") [17].

Below Result 3.1 is combined with Theorem 2.1 to extend Result 3.1 to topological properties $Q$ for which $P($ Urysohn) and $P 3$ exist.

Theorem 3.4. Let $Q$ be a topological property for which $Q($ Urysohn) exists and let $(X, T)$ be a space. Then $(X, T)$ is ((weakly Q(Urysohn)) and "not-T" $T_{0}$ ") iff for each OXTO subset $Y$ of $(X, T),\left(Y, T_{Y}\right)$ is a maximal, proper, dense, $Q($ Urysohn) subspace of $(X, T)$.

Proof. Suppose $(X, T)$ is (weakly $Q\left(\right.$ Urysohn) and "not- $T_{0}$ ”). Then $(X, T)$ is (weakly $(Q($ Urysohn $)) o$ and "not- $T_{0}$ ") and for each OXTO subset $Y$ of $(X, T),\left(Y, T_{Y}\right)$ is a maximal, proper, dense, (Q(Urysohn))o subspace of $(X, T)$. Thus, for each $O X T O$ subset $Y$ of $(X, T),\left(Y, T_{Y}\right)$ is a maximal, proper, dense, $Q($ Urysohn) subspace of $(X, T)$.

Conversely, suppose $(X, T)$ is a space such that for each OXTO subset $Y$ of $(X, T),\left(Y, T_{Y}\right)$ is a maximal, proper, dense, $Q$ (Urysohn) subspace of $(X, T)$. Then for each $O X T O$ subset $Y$ of $(X, T),\left(Y, T_{Y}\right)$ is a maximal, proper, dense, $(Q($ Urysohn $)) o$ subspace of $(X, T)$, which implies $(X, T)$ is (weakly $(Q($ Urysohn $)) o$ and "not- $T_{0}$ ”), which equals ((weakly $Q($ Urysohn $))$ and "not- $T_{0}$ ”). 
Theorem 3.5. Let $Q$ be a topological property for which $Q 3$ exists and let $(X, T)$ be a space. Then $(X, T)$ is (weakly $Q 3$ and "not- $T_{0}$ ") iff for each OXTO subset $Y$ of $(X, T),\left(Y, T_{Y}\right)$ is a maximal, proper, dense, Q3 subspace of $(X, T)$.

The proof is similar to that of Theorem 3.4 and is omitted.

\section{References}

[1] A. S. Davis, Indexed systems of neighborhoods for general topological spaces, Amer. Math. Monthly 68(9) (1961), 886-893.

DOI: http://dx.doi.org/10.2307/2311686

[2] C. Dorsett, Weakly $P$ properties, Fundamental J. Math. Math. Sci. 3(1) (2015), 83-90.

[3] C. Dorsett, Complete characterization of weakly $P o$ and related spaces and properties, J. Math. Sci.: Adv. Appl. 45 (2017), 97-109.

DOI: http://dx.doi.org/10.18642/jmsaa_7100121834

[4] C. Dorsett, Pluses and needed changes in topology resulting from additional properties, Far East Journal of Mathematical Sciences 101(4) (2017), 803-811.

DOI: http://dx.doi.org/10.17654/MS101040803

[5] C. Dorsett, Weakly $P$ corrections and new, fundamental topological properties and facts, Fundamental J. Math. Math. Sci. 5(1) (2016), 11-20.

[6] C. Dorsett, $T_{0}$-identification $P$ and weakly $P$ properties, Pioneer J. Math. Math. Sci. 15(1) (2015), 1-8.

[7] C. Dorsett, Weakly $P 1$, weakly $P o$, and $T_{0}$-identification properties, Fundamental J. Math. Math. Sci. 6(1) (2016), 33-43.

[8] C. Dorsett, Weakly P2 and related properties, Fundamental J. Math. Math. Sci. 4(1) (2015), 11-21.

[9] C. Dorsett, Weakly $P($ Urysohn $)$ spaces and properties, and equivalent separation axioms, Fundamental J. Math. Math. Sci. 6(2) (2016), 99-110.

[10] C. Dorsett, The equivalence of $T_{0}, T_{1}, T_{2}$ Urysohn, and $T_{3}$ and infinitely many new characterizations of the $T_{3}$ property, J. Math. Sci.: Adv. Appl. 42 (2016), 13-26.

DOI: http://dx.doi.org/10.18642/jmsaa_7100121732

[11] C. Dorsett, Generalized Urysohn spaces, Revista Colombiana de Math. 22 (1988), 149-160. 
[12] C. Dorsett, The complete characterization and internalization of weakly $P 1$, and additional insights for weakly $P o$ and weakly $P 1$ properties, accepted by Pioneer Journal of Mathematics and Mathematical Sciences.

[13] C. Dorsett, Characterization and internalization of weakly $P 2$, and insights for weakly $P o$, weakly $P 1$ and weakly $P 2$ spaces, accepted by the Far East Journal of Mathematical Sciences.

[14] C. Dorsett, New characterizations of topological product properties weakly $P($ Urysohn) spaces and properties, submitted.

[15] C. Dorsett, Weakly $P($ Urysohn) spaces and properties, and equivalent separation axioms, Fundamental J. Math. Math. Sci. 6(2) (2016), 99-110.

[16] C. Dorsett, Additional properties for weakly Po and related properties with an application, J. Math. Sci.: Adv. Appl. 47 (2017), 53-64.

DOI: http://dx.doi.org/10.18642/jmsaa_7100121877

[17] C. Dorsett, Additional properties and characterizations of $T_{0}$, Qo, weakly Qo, and maximal, proper, dense, Qo OXTO subspaces in weakly $Q o$ and "not- $T_{0}$ " spaces, J. Math. Sci.: Adv. Appl. 49 (2018), 15-28.

DOI: http://dx.doi.org/10.18642/jmsaa_7100121916

[18] W. Dunham, Weakly Hausdorff spaces, Kyungpook Math. J. 15(1) (1975), 41-50.

[19] N. Shanin, On separations in topological spaces, C. R. (Doklady) Acad. Sci. URSS (N.S.) 38 (1943), 110-113.

[20] M. Stone, Application of Boolean Algebras to Topology, Mat. Sb. 1 (1936), 765-771.

[21] P. Urysohn, Uber die machtigkeit der zusammenhangenden mengen, Math. Ann. 94(1) (1925), 262-295.

DOI: https://doi.org/10.1007/BF01208659

[22] L. Vietoris, Stetige Mengen, Monatshefte für Mathematik und Physik 31(1) (1921), 173-204.

DOI: https://doi.org/10.1007/BF01702717 